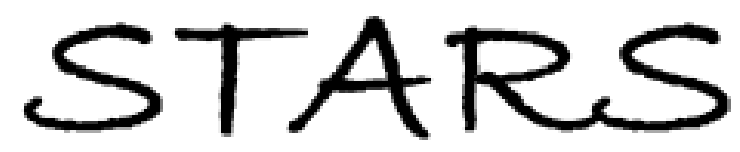

University of Central Florida

STARS

$1-1-2011$

\title{
Vortex motion in superfluid He-4: Reformulation in the extrinsic vortex-filament coordinate space
}

Bhimsen K. Shivamoggi

Find similar works at: https://stars.library.ucf.edu/facultybib2010 University of Central Florida Libraries http://library.ucf.edu

This Article is brought to you for free and open access by the Faculty Bibliography at STARS. It has been accepted for inclusion in Faculty Bibliography 2010 s by an authorized administrator of STARS. For more information, please contact STARS@ucf.edu.

\section{Recommended Citation}

Shivamoggi, Bhimsen K., "Vortex motion in superfluid He-4: Reformulation in the extrinsic vortex-filament coordinate space" (2011). Faculty Bibliography 2010s. 1913.

https://stars.library.ucf.edu/facultybib2010/1913

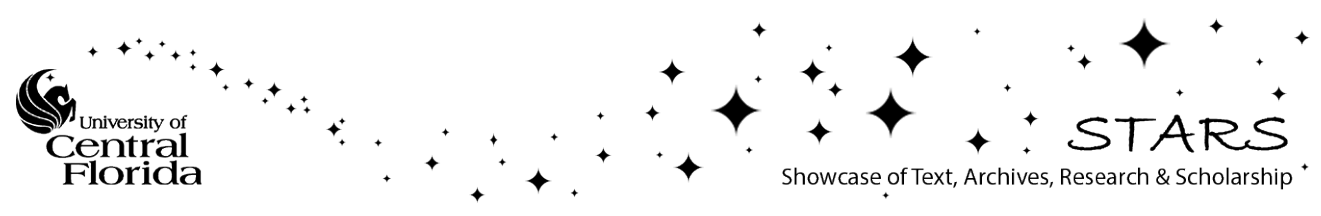




\title{
Vortex motion in superfluid ${ }^{4} \mathrm{He}$ : Reformulation in the extrinsic vortex-filament coordinate space
}

\author{
Bhimsen K. Shivamoggi* \\ J. M. Burgers Centre and Fluid Dynamics Laboratory, Department of Physics, Eindhoven University of Technology, \\ NL-5600MB Eindhoven, The Netherlands
}

(Received 22 September 2010; revised manuscript received 16 December 2010; published 21 July 2011)

\begin{abstract}
Vortex motion in superfluid ${ }^{4} \mathrm{He}$ is considered by reformulating the Hall-Vinen equation (originally formulated in the intrinsic geometric parameter space) in the extrinsic vortex filament coordinate space which is shown to provide a useful alternative approach. It provides insights into the physical implications of one aspect of the friction term, associated with the friction coefficient $\alpha$, in the Hall-Vinen notation, as well as the previous neglect of the other aspect, associated with the friction coefficient $\alpha^{\prime}$. A nonlinear Schrodinger equation for the propagation of nonlinear Kelvin waves on a vortex filament in a superfluid is given. The localized vortex kink solution of this equation is shown to be driven unstable by the normal fluid flow along the vortex in qualitative similarity with the Donnelly-Glaberson instability of Kelvin waves on a vortex. This instability is demonstrated in a more clear way by considering the problem of a rotating planar vortex filament in a superfluid. Though the friction term associated with $\alpha$, for very small $\alpha$, has little capacity to make significant contribution to the vortex motion in a quantitative way, it appears to be able to influence the vortex kink characteristics in a qualitative way.
\end{abstract}

DOI: 10.1103/PhysRevB.84.012506

The Landau ${ }^{1}$ model of liquid ${ }^{4} \mathrm{He}$ considers the superfluid below the $\lambda$-point as an inviscid, irrotational "background fluid" with thermal excitations (the phonons and rotons) moving upon that background. These excitations constitute the normal fluid which would not interact with the superfluid except for the presence of vortices. ${ }^{2}$ The vortices scatter the thermal excitations when there is a relative velocity between them, thus generating the so-called "mutual friction" (Feynman). ${ }^{6}$ The existence of mutual friction ${ }^{7}$ was confirmed by the experiment on the attenuation of second sound in uniformly rotating liquid ${ }^{4} \mathrm{He}$ (Hall and Vinen). ${ }^{9,10}$ These experiments, on assuming that a thermal excitation can exchange momentum only in a direction perpendicular to the scattering vortex, then indicated (Vinen) ${ }^{11}$ that the friction force will have a constant and large value for all directions of the relative velocity perpendicular to the rotation axis (with which the vortices are aligned) but will be zero for directions parallel to the latter. On the other hand, vortices in liquid ${ }^{4} \mathrm{He}$ have a core radius of the order of the quantum coherence length $(1 \AA)$, so the detailed core physics is not very relevant to the dynamical effects of the vortex away from the core. So, barring vortex reconnection events [which involve sharp distortions of vortex lines (Paoletti et al. $)^{12}$ and the concomitant generation of Kelvin waves (Yepez et al. $),{ }^{13}$ vortices in superfluid ${ }^{4} \mathrm{He}$ behave like classical vortex filaments, the only difference being that their circulations and core radii exhibit quantum mechanical features. Indeed, the pioneering numerical simulations of Schwarz, ${ }^{14,15}$ on superfluid vortex motion dynamics, are based on this idea.

The leading-order behavior of the vortex-induced flow velocity formula in classical fluid dynamics is given by the so-called local induction approximation (LIA) (Da Rios ${ }^{16}$ and Arms and $\mathrm{Hama}^{17}$ ) which resolves the singularity due to the neglect of the finite vortex core size by an asymptotic calculation. Using the LIA, Da Rios ${ }^{16}$ and Betchov ${ }^{18}$ derived a set of coupled equations governing the inextensional motion of a vortex filament in an irrotational fluid in terms of time evolution of its intrinsic geometric parameters - curvature and torsion. Hasimoto $^{19}$ showed that Da Rios-Betchov equations can be
PACS number(s): 67.25.dk

elegantly combined to give a nonlinear Schrodinger equation. The single-soliton solution of this equation (Zakharov and Shabat ${ }^{20}$ provides a description of an isolated loop of helical twisting motion along the vortex line. The LIA is, however, hampered by the fact that the motion of the vortex filament is assumed to be governed solely by the local features on the filament, so distant parts of the filament need to remain sufficiently separated during the motion. This is violated by the large-amplitude solutions in LIA as in a self-interaction of the vortex filament.

Interestingly, vortices in superfluid ${ }^{4} \mathrm{He}$ appear to be better suited than those in ordinary fluids for the application of LIA, because the thin cores of vortices validate the asymptotic evaluation implicit in the LIA which holds in the limit of vanishingly small vortex core size, while interactions between different segments of a vortex materialize only when the distance between them is of the order of few angstroms. ${ }^{14,15}$

Upon including the effect of the frictional force exerted by the normal fluid on a vortex, the self-induced velocity of the vortex in the reference frame moving with the superfluid according to the LIA is given by the Hall-Vinen equation ${ }^{9,10}$

$$
\begin{aligned}
\mathbf{v}= & \gamma \kappa \hat{\mathbf{t}} \times \hat{\mathbf{n}}+\alpha \hat{\mathbf{t}} \times(\mathbf{U}-\gamma \kappa \hat{\mathbf{t}} \times \hat{\mathbf{n}}) \\
& -\alpha^{\prime} \hat{\mathbf{t}} \times[\hat{\mathbf{t}} \times(\mathbf{U}-\gamma \kappa \hat{\mathbf{t}} \times \hat{\mathbf{n}})] .
\end{aligned}
$$

Here, $\mathbf{U}$ is the normal fluid velocity taken to be constant in space and time and prescribed ${ }^{21}$ (see, e.g., Refs. 14 and 15), $\kappa$ is the average curvature, and $\hat{\mathbf{t}}$ and $\hat{\mathbf{n}}$ are unit tangent and normal vectors, respectively, to the vortex filament, and $\gamma=\Gamma \ln \left(c / \kappa a_{0}\right)$, where $\Gamma$ is the quantum of circulation, $c$ is a constant of order 1 , and $a_{0} \approx 1.3 \times 10^{-8} \mathrm{~cm}$ is the effective core radius of the vortex. $\alpha$ and $\alpha^{\prime}$ are the friction coefficients which are usually found to be small (except near the $\lambda$-point) so the short-term vortex motion appears to be only weakly affected by the friction. However, the friction term associated with $\alpha$ plays the dual roles of driving force and drag force. ${ }^{14,15}$ It can therefore lead to both growth and decay of the vortex length and hence can produce important qualitative effects. On the other hand, the friction term associated with $\alpha^{\prime}$ arises partly from the asymmetry in the fundamental roton-vortex 
scattering and partly from the Magnus effect and is usually dropped under the pretext that $\alpha>\alpha^{\prime}$ (Ref. 22) (Vinen and Niemela). ${ }^{23}$ Nevertheless, determination of the vortex motion from the Hall-Vinen equation (1), which is formulated in the intrinsic geometric parameter space, is a highly nontrivial task and numerical simulations have been so far essentially the only method of investigation. In this paper, we will show that a reformulation of the Hall-Vinen equation (1) in the extrinsic vortex filament coordinate space provides a useful alternative approach in this regard-it provides insight into the fundamental importance of the friction term associated with $\alpha$ as well as the previous neglect of the friction term associated with $\alpha^{\prime}$.

The extrinsic vortex filament coordinate space formulation considers only small-amplitude vortex motions in the LIA model. So it avoids the problems besetting the large-amplitude solutions in LIA.

Consider the vortex essentially aligned along the $x$-axis (Dmitreyev ${ }^{24}$ and Shivamoggi and van Heijst ${ }^{25}$ ) and take $\mathbf{U}=$ $U_{1} \hat{\mathbf{i}}_{x}$; Eq. (1) then becomes

$\mathbf{v}=\left(1-\alpha^{\prime}\right) \gamma \kappa \hat{\mathbf{t}} \times \hat{\mathbf{n}}+\alpha \hat{\mathbf{t}} \times \mathbf{U}+\alpha \gamma \kappa \hat{\mathbf{n}}-\alpha^{\prime} U_{1} \hat{\mathbf{t}}+\alpha^{\prime} U_{1} \hat{\mathbf{i}}_{x}$.

We assume the deviations from the $x$-axis to be small,

$$
\mathbf{r}=x \hat{\mathbf{i}}_{x}+y(x, t) \hat{\mathbf{i}}_{y}+z(x, t) \hat{\mathbf{i}}_{z} .
$$

On noting,

$$
\begin{aligned}
\hat{\mathbf{v}} \equiv \frac{d \mathbf{r}}{d t}=y_{t} \hat{\mathbf{i}}_{y}+z_{t} \hat{\mathbf{i}}_{z} \\
\hat{\mathbf{t}} \equiv \frac{d \mathbf{r}}{d s} \approx\left(\hat{\mathbf{i}}_{x}+y_{x} \hat{\mathbf{i}}_{y}+z_{x} \hat{\mathbf{i}}_{z}\right)\left[1-\frac{1}{2}\left(y_{x}^{2}+z_{x}^{2}\right)\right], \\
\kappa \hat{\mathbf{n}} \equiv \frac{d \hat{\mathbf{t}}}{d s} \approx-\left(y_{x} y_{x x}+z_{x} z_{x x}\right)\left[1-\frac{1}{2}\left(y_{x}^{2}+z_{x}^{2}\right)\right] \hat{\mathbf{i}}_{x} \\
+\left[y_{x x}\left\{1-\frac{1}{2}\left(y_{x}^{2}+z_{x}^{2}\right)\right\}\right. \\
\left.-\left(y_{x}^{2} y_{x x}+y_{x} z_{x} z_{x x}\right)\right]\left[1-\frac{1}{2}\left(y_{x}^{2}+z_{x}^{2}\right)\right] \hat{\mathbf{i}}_{y} \\
+\left[z_{x x}\left\{1-\frac{1}{2}\left(y_{x}^{2}+z_{x}^{2}\right)\right\}\right. \\
\left.-\left(z_{x} y_{x} y_{x x}+z_{x}^{2} z_{x x}\right)\right]\left[1-\frac{1}{2}\left(y_{x}^{2}+z_{x}^{2}\right)\right] \hat{\mathbf{i}}_{z}
\end{aligned}
$$

and putting,

$$
\Phi \equiv y+i z
$$

and keeping only linear terms associated with $\alpha$ and $\alpha^{\prime}$ (since $\alpha$ and $\alpha^{\prime}$ are very small) ${ }^{22} \mathrm{Eq}$. (2) leads to the nonlinear Schrodinger equation

$$
\begin{aligned}
& \frac{1}{i}\left(\Phi_{t}+\alpha^{\prime} U_{1} \Phi_{x}\right)+\alpha U_{1} \Phi_{x} \\
& \quad=\left(1-\alpha^{\prime}\right) \gamma \Phi_{x x}-i \alpha \gamma \Phi_{x x}-\frac{3 \gamma}{2}\left|\Phi_{x}^{2}\right| \Phi_{x x}
\end{aligned}
$$

which describes the propagation of nonlinear Kelvin waves on a vortex in a superfluid. It may be noted that the friction term associated with $\alpha^{\prime}$ on the left-hand side in Eq. (8) can be transformed away via the Galilean transformation

$$
q(x, t) \Rightarrow q(\xi, t), \quad \xi \equiv x-\alpha^{\prime} U_{1} t,
$$

while the friction term associated with $\alpha^{\prime}$ on the right-hand side can be eliminated by renormalizing the vortex strength $\gamma,{ }^{26}$ albeit an $O\left(\alpha \alpha^{\prime}\right)$ correction to the second term on the right-hand side which is small [compared with $O(\alpha)$ ], and an $O\left(\alpha^{\prime}\right)$ correction to the nonlinear term on the right-hand side which is also to be neglected because only linear terms associated with $\alpha$ and $\alpha^{\prime}$ are to be kept (as mentioned before). Alternatively, one may just drop the friction term associated with $\alpha^{\prime}$ on the right-hand side in Eq. (8) on the grounds that $\alpha^{\prime}$ is very small compared with 1 and hence is of no qualitative significance unlike the friction term associated with $\alpha$. This provides some insight into the neglect of the friction term associated with $\alpha^{\prime}$, as in Refs. 14 and 15 .

Look for a nonlinear localized Kelvin stationary wave solution

$$
\Phi(x, t)=\nu \psi(\xi-u \gamma t) e^{i(\sigma \xi-c \gamma t)+\mu t}
$$

and assume $\psi$ is slowly varying, so only linear terms in the derivatives $\psi^{\prime}$ and $\psi^{\prime \prime}$ are kept while the $\psi^{\prime}$ and $\psi^{\prime \prime}$ terms associated with $\alpha$ and $\alpha^{\prime}$ are dropped altogether (since $\alpha$ and $\alpha^{\prime}$ are very small $)^{22}$; we then obtain from Eq. (8),

$$
\begin{aligned}
& \psi^{\prime \prime}+i(2 \sigma-u) \psi^{\prime}+\left[c-(1-i \alpha) \sigma^{2}\right. \\
& \left.-i \alpha \sigma U_{1}+i \mu\right] \psi+\frac{3 v \sigma^{4}}{2} \psi^{3} e^{2 \mu t}=0 .
\end{aligned}
$$

We now put

$$
\sigma=\frac{u}{2}, \beta \equiv \sigma^{2}-c, \mu=\frac{\alpha u}{2}\left(U_{1}-\frac{u}{2}\right)
$$

and drop again nonlinear terms associated with $\alpha$. The first relation implies that the velocity of propagation of the structure is twice the torsion, such as the case with Hasimoto's ${ }^{19}$ solution in ordinary fluid. Further, $\beta$ is a measure of the curvature. Equation (11) then becomes

$$
\psi^{\prime \prime}-\beta \psi+\frac{3 v \sigma^{4}}{2} \psi^{3}=0
$$

which admits a solitary-wave solution

$$
\psi=\sqrt{\frac{4 \beta}{3 v \sigma^{4}}} \operatorname{sech} \sqrt{\beta}(\xi-2 \sigma \gamma t)
$$

describing a propagating damped Kelvin kink on a vortex in superfluid ${ }^{4} \mathrm{He}$. Observe that the damping parameter $\mu$ vanishes when

(i) either $\alpha=0$,ordinary fluid case,

(ii) or $u=2 U_{1}$, special superfluid case.

Further, the damping is symmetric with respect to the direction of propagation of the vortex kink (as it should be)-this symmetry is, however, broken by the normal fluid velocity component $U_{1}$ along the vortex. The vortex kink growth associated with the latter aspect has interesting qualitative similarities with the Donnelly-Glaberson instability (Cheng et $a l^{28}$ and Glaberson et $a .^{29}$ ) of Kelvin waves on a vortex 
driven by the normal fluid velocity component along the vortex.

In the second case, namely, Eq. (15a), the vortex kink is undamped - the nonlinearity in the system, under condition (15b), balances both the dispersion and the mutual friction and the vortex kink amplitude and its propagation speed are determined by the normal fluid velocity $U_{1}$. Thus, even though the friction term associated with $\alpha$, for very small $\alpha$, has little capacity to make significant contribution to the vortex motion in a quantitative way, it appears to be able to change vortex kink dynamics characteristics in a qualitative way. This feature reveals itself in a more striking way on considering Hasimoto's ${ }^{30}$ rotating-planar vortex problem in a superfluid, as seen below.

Consider a planar vortex filament with shape $y=y(x)$ lying in the $x, y$ plane which is rotated with a uniform angular velocity $\Omega$ about the $x$-axis.

On noting that $\hat{\mathbf{t}}$ now has no $z$-component because the vortex filament in this case is restricted to be planar, so $z_{x}=0$, we have

$$
\begin{gathered}
\hat{\mathbf{t}}=\left\langle 1, y_{x}, 0\right\rangle \frac{1}{\sqrt{1+y_{x}^{2}}}, \\
\kappa \hat{\mathbf{n}} \equiv \frac{d \hat{\mathbf{t}}}{d s}=\left\langle-\frac{y_{x} y_{x x}}{\left(1+y_{x}^{2}\right)^{3 / 2}}, \frac{y_{x x}}{\left(1+y_{x}^{2}\right)^{3 / 2}}, 0\right\rangle \frac{1}{\sqrt{1+y_{x}^{2}}},
\end{gathered}
$$

and taking $\mathbf{U}=U_{1} \hat{\mathbf{i}}_{x}$, the $z$-component of Eq. (2) leads to

$$
\left(1-\alpha^{\prime}\right) \gamma \frac{y_{x x}}{\left(1+y_{x}^{2}\right)^{3 / 2}}-\alpha U_{1} \frac{y_{x}}{\left(1+y_{x}^{2}\right)^{1 / 2}}=-\Omega y .
$$

Note that the vortex is rotating in the retrograde sense (i.e., opposite to that of the vortex core). Further, the friction term associated with $\alpha^{\prime}$ on the left-hand side can again be eliminated by renormalizing the vortex strength $\gamma$.

Putting,

$$
y_{x}=\tan \theta,
$$

$\theta$ being the angle between the tangent to the vortex and the $x$-axis, Eq. (18) becomes

$$
\gamma \frac{d^{2} \theta}{d s^{2}}-\alpha U_{1} \cos \theta \cdot \frac{d \theta}{d s}+\Omega \sin \theta=0 .
$$

Equation (20) indicates a vortex growth via a normal-fluid flow-driven instability ${ }^{31}$ and confirms that the friction term associated with $\alpha$ plays the dual roles of driving force and drag force ${ }^{14,15}$ and, hence, leads to both growth and decay of the vortex line length. Indeed, assuming $|\Omega / \gamma| \ll 1$ and expanding in powers of $\theta$, Eq. (20) can be approximated by

$$
\gamma \frac{d^{2} \theta}{d s^{2}}+\alpha U_{1}\left(\frac{\theta^{2}}{2}-1\right) \frac{d \theta}{d s}+\Omega \theta=0
$$

which is just the van der Pol equation. If the normal-fluid velocity is in the same direction as that of vorticity in the undisturbed vortex, then there is decay/growth of the vortex length if $|\theta|^{2} \gtrless 4$ and vice versa if the normal-fluid velocity is in the direction opposite to that of vorticity in the undisturbed vortex. The friction term associated with $\alpha$ appears again to be able to change the vortex motion aspects in a qualitative way.

Beginning with the work of Feynman, ${ }^{6}$ the phenomenological model of quantized vortices as classical vortex filaments subject to an effective frictional force (simulating interactions with thermal excitations) has been the standard approach to investigate vortex dynamics in superfluid ${ }^{4} \mathrm{He}$. The theoretical formulations developed in this paper provide insight into the fundamental importance of the friction term associated with the friction coefficient $\alpha$ as well as the previous neglect of the other friction term associated with the friction coefficient $\alpha^{\prime}$. Further, though the friction term associated with $\alpha$, for very small $\alpha$, has little capacity to make significant contribution to the vortex motion in a quantitative way, it appears to be able to influence the vortex kink characteristics in a qualitative way. This becomes possible because of the ability of this friction term to play the dual roles of driving force and drag force.

This work was carried out when the author held a visiting research appointment at the Eindhoven University of Technology supported by a Grant from The Netherlands Organization for Scientific Research (NWO). The author is thankful to Gert Jan van Heijst for his hospitality and helpful discussions. The author is thankful to K. R. Sreenivasan, Dan Lathrop, and Yuki Sato for helpful remarks and Michael Johnson for helpful discussions. The author is very indebted to the referees for their helpful remarks and criticism.
*Permanent address: University of Central Florida, Orlando, Florida 32816-1364, USA.

${ }^{1}$ L. D. Landau, J. Phys. (USSR) 5, 71 (1941).

${ }^{2}$ Vorticity in superfluid ${ }^{4} \mathrm{He}$ is confined to vortices which are topological linear defects with the superfluid density vanishing at the vortex core. The circulation around a vortex is quantized (Onsager), ${ }^{3}$ which was confirmed in an ingenious experiment by Vinen. ${ }^{4}$ Direct observation of vortex cores was accomplished (Bewley et al. $)^{5}$ by using small solid hydrogen particles as traces in liquid ${ }^{4} \mathrm{He}$.

${ }^{3}$ L. Onsager, Proceedings of International Conference on Theoretical Physics (Science Council of Japan, Tokyo, 1953), p. 877.

${ }^{4}$ W. F. Vinen, Proc. R. Soc. London A 260, 218 (1961).
${ }^{5}$ G. P. Bewley, D. P. Lathrop and K. R. Sreenivasan, Nature 441, 588 (2006).

${ }^{6}$ R. P. Feynman, Prog. Low Temp. Phys. 1, 17 (1955).

${ }^{7}$ Due to our poor understanding of the microscopic origin of the physical processes underlying the mutual friction (Donnelly ${ }^{8}$ ), an adequate theory of the fundamental roton-vortex scattering process in superfluid ${ }^{4} \mathrm{He}$ is not at hand yet.

${ }^{8}$ R. J. Donnelly, Quantized Vortices in Helium II (Cambridge University Press, Cambridge, 1991), p. 86.

${ }^{9}$ H. E. Hall and W. F. Vinen, Proc. R. Soc. London A 238, 204 (1956)

${ }^{10}$ H. E. Hall and W. F. Vinen, Proc. R. Soc. London A 238, 215 (1956).

${ }^{11}$ W. F. Vinen, in Quantum Fluids, edited by D. F. Brewer (NorthHolland, Amsterdam, 1966), p. 74. 
${ }^{12}$ M. S. Paoletti, M. E. Fisher, and D. P. Lathrop, Physica D 239, 1367 (2010).

${ }^{13}$ J. Yepez, G. Vahala, L. Vahala, and M. Soe, Phys. Rev. Lett. 103, 084501 (2009).

${ }^{14}$ K. W. Schwarz, Phys. Rev. B 31, 5782 (1985).

${ }^{15}$ K. W. Schwarz, Phys. Rev. B 38, 2398 (1988).

${ }^{16}$ L. S. Da Rios, Rend. Circ. Mat. Palermo 22, 117 (1906).

${ }^{17}$ R. J. Arms and F. R. Hama, Phys. Fluids 8, 553 (1965).

${ }^{18}$ R. Betchov, J. Fluid Mech. 22, 471 (1965).

${ }^{19}$ H. Hasimoto, J. Fluid Mech. 51, 477 (1972).

${ }^{20}$ V. E. Zakharov and A. B. Shabat, Sov. Phys. JETP 34, 62 (1972).

${ }^{21}$ This formulation is kinematical-the effect of the vortices on the normal fluid is ignored.

${ }^{22}$ Actual numerical values of $\alpha$ and $\alpha^{\prime}$ at a couple of typical temperatures taken from table 1 of $\mathrm{Schwarz}^{14}$ are

$$
T(K)=1.0, \alpha=0.005, \alpha^{\prime}=0.003
$$$$
T(K)=1.5, \alpha=0.073, \alpha^{\prime}=0.018 \text {. }
$$

${ }^{23}$ W. F. Vinen and J. J. Niemela, J. Low Temp. Phys. 128, 167 (2002).

${ }^{24}$ V. P. Dmitreyev, Am. J. Phys. 73, 563 (2005)

${ }^{25}$ B. K. Shivamoggi and G. J. F. van Heijst, Phys. Lett. A 374, 1742 (2010).

${ }^{26}$ This result is also apparent in the vortex-motion formulation via the Gross-Pitaevskii theory (Pismen). ${ }^{27}$

${ }^{27}$ L. M. Pismen, Vortices in Nonlinear Fields (Clarendon Press, Oxford, 1999), p. 166.

${ }^{28}$ D. K. Cheng, M. W. Cromar, and R. J. Donnelly, Phys. Rev. Lett. 31, 433 (1973).

${ }^{29}$ W. I. Glaberson, W. W. Johnson, and R. M. Ostermeier, Phys. Rev. Lett. 33, 1197 (1974).

${ }^{30}$ H. Hasimoto, J. Phys. Soc. Jpn. 31, 293 (1971).

${ }^{31}$ Experiments by Sato et al. ${ }^{32}$ on vortex dynamics in liquid ${ }^{4} \mathrm{He}$ near $T_{\lambda}$ indicated vortex growth due to an instability.

${ }^{32}$ Y. Sato, A. Joshi, and R. Packard, Phys. Rev. B 76, 052505 (2007). 